\title{
De la réanimation du sujet âgé à la création de filières réa-gériatriques
}

\section{Lifelines of Intensive Care Medicine in Elderly Patients}

\author{
H. Vallet $\cdot$ J. Cohen-Bittan $\cdot$ J. Boddaert \\ (C) SRLF et Lavoisier SAS 2015
}

D'après le rapport de la dernière Assemblée mondiale sur le vieillissement, la population âgée de plus de 60 ans a triplé au cours des 50 dernières années et triplera encore à l'horizon 2050 [1]. Le groupe d'âge qui progresse le plus rapidement est celui des personnes de 80 ans et plus, avec une augmentation de $3,8 \%$ par an. D'ici 2050, un cinquième des personnes âgées aura 80 ans ou plus. La réanimation est concernée par cette évolution démographique. On assiste en effet à une augmentation du nombre d'admissions de patients âgés de plus de 80 ans dans ces services sur les 15 dernières années [2,3]. En parallèle, le nombre de publications scientifiques sur le sujet est en constante augmentation [4].

Or l'impact du vieillissement de la population pose pour l'hôpital un certain nombre de questions, tant médicales qu'éthiques ou économiques, qui se reflètent tout au long du parcours de soins hospitaliers des patients âgés. Il pose aussi la question de la place de la gériatrie dans la prise en charge des patients âgés. Tous les patients âgés ne doivent - ni ne peuvent - aller en gériatrie. Cependant, si l'expertise gériatrique aiguë peut être définie par la gestion de la multimorbidité dans le cadre d'une pathologie aiguë, en préservant l'autonomie et la qualité de vie, il est important de lui trouver une juste place au sein de ce parcours hospitalier.

Il serait illusoire de dire que la gériatrie a largement démontré son efficacité dans la prise en charge des patients âgés. Par exemple, l'évaluation gériatrique standardisée n'a montré qu'un intérêt limité pour les patients. Mais c'est en collaborant avec les autres spécialités qu'elle a pu montrer son efficacité et qu'elle devra trouver une place supplémentaire dans l'organisation hospitalière. Ainsi, l'orthogériatrie, née dans les années 1950, qui repose sur une implica-

H. Vallet $\cdot$ J. Cohen-Bittan $\cdot$ J. Boddaert $(\bowtie)$

Unité périopératoire gériatrique,

groupe hospitalier (GH) Pitié-Salpêtrière,

Assistance Publique-Hôpitaux de Paris (APHP), Paris, France ;

Sorbonne Universités UPMC Univ Paris 06, DHU FAST

e-mail : jacques.boddaert@aphp.fr tion précoce des gériatres, met en évidence la nécessité et le bénéfice de leur confier la gestion de la multimorbidité dans ce cadre. La première filière d'orthogériatrie française, créée en 2009 autour de l'Unité périopératoire de gériatrie (UPOG) du groupe hospitalier Pitié Salpêtrière, est un exemple d'approche de parcours de soins. Avant son ouverture, les urgentistes, anesthésistes, orthopédistes, gériatres d'aigu et d'aval en soins de suite et réadaptation (SSR) ont élaboré des critères et objectifs communs. Quatre facteurs principaux de la prise en charge ont été identifiés : 1) aux urgences, alerte immédiate devant toute fracture de l'extrémité supérieure du fémur ; 2) urgence chirurgicale à opérer le plus tôt possible (chirurgie $24 \mathrm{~h} / 24 \mathrm{~h}$ pour un délai chirurgical cible $<48 \mathrm{~h})$; 3) transfert rapide en UPOG dès la fin de la chirurgie ( $<48$ heures) ; 4) transfert rapide des patients dans un SSR adapté. La prise en charge en UPOG intervenait dès la sortie du bloc opératoire, sans séjour en chirurgie, et faisait intervenir l'ensemble de l'équipe médicale gériatrique et paramédicale (infirmière, aide-soignant, kinésithérapeute, ergothérapeute, psychologue, diététicienne et assistante sociale), afin de gérer au plus tôt les comorbidités, de mobiliser rapidement les patients (fauteuil dans les $24 \mathrm{~h}$, marche dans les $24-48$ heures de leur arrivée) et de prendre en charge les complications postopératoires. Par rapport à une prise en charge standard, la filière UPOG a ainsi amélioré la morbidité (transferts en réanimation, escarres), ainsi que la mortalité et les réadmissions à six mois [5].

En raison de ces données démographiques et organisationnelles, la création de filières de réa-gériatrie structurée devrait n'être qu'une question de temps. La mortalité du patient âgé en réanimation, notamment après 80 ans, est élevée. En fonction des études, elle varie de 29 à $38 \%$ au sein de la réanimation [6,7], de 28 à $41 \%$ dans le mois qui suit le passage en réanimation $[8,9]$ et de 46 à $69 \%$ un an après le séjour en réanimation $[7,8]$. Cette mortalité est particulièrement importante dans les premières semaines qui suivent l'admission en réanimation, semaines où l'expertise gériatrique doit trouver sa place en aval de la réanimation, pour la gestion de cette multimorbidité et la prévention des complications propres à ces patients âgés. Or, si des organisations 
existent, aucune n'a fait l'objet d'une publication scientifique. Le travail sur la fluidité du parcours de soins reste donc à faire, centré autour de la réanimation, en associant les urgentistes, les SAMU, les réanimateurs, les gériatres jusqu'aux services de Soins de suite et réadaptation, et en travaillant sur des questions précises, telles que :

- l'adaptation des techniques propres à la réanimation chez le sujet âgé, comme la réflexion autour de l'utilisation des hypnotiques par exemple. L'essentiel des facteurs pronostiques relève de l'expertise de réanimation, comme le type d'admission (réanimation médicale, chirurgicale d'urgence ou chirurgicale programmée) [7], la gravité du patient à l'admission (évaluée par le SAPS ou le SOFA) $[7,8]$ ou encore la décision de limitation thérapeutique à l'admission du malade $[8,10]$;

- l'accès à la réanimation des patients âgés, qui reste un sujet éthique et difficile comme le montrent les travaux du groupe ICE-CUB [11]. L'identification des patients âgés capables d'aller en réanimation doit reposer sur des outils ou procédures d'évaluation objectifs et faisables dans l'urgence, permettant de mieux préciser ce qui revient à l'âge (vulnérabilité physiologique) et à la multimorbidité (cinq à sept pathologies en moyenne), mais prenant en compte également le statut fonctionnel, nutritionnel, voire cognitif. Ainsi, anticiper les capacités de récupération du sujet âgé, tant sur le plan physique que fonctionnel et cognitif, est un enjeu majeur pour envisager un transfert en réanimation;

- la meilleure organisation de la prise en charge, au décours immédiat de la réanimation, des comorbidités, des complications éventuelles, et la mise en route d'une réhabilitation fonctionnelle précoce aussi urgente que les soins médicaux, au sein de filières de soins organisées. Car si l'âge en lui-même est associé au pronostic en réanimation [8], le poids des comorbidités et du statut fonctionnel préhospitalier est également déterminant $[10,12]$, à côté des facteurs liés à la pathologie aiguë et à la réanimation. En ce sens, l'approche par la création de parcours de soins et de filières pourrait améliorer la prise en charge de ces patients âgés, en associant les compétences de chacun.

Mais devant toutes ces difficultés, faut-il vraiment ouvrir la réanimation aux patients âgés ? De surcroît avec multimorbidité ? Il semble judicieux de faciliter l'accès à la réanimation des « bons » patients âgés dans le cadre d'un projet de soins « cohérent », où chaque mot a son importance et appelle une précision. « Bons », car une évaluation objective, fiable, faisable et souvent pluridisciplinaire a jugé que le patient âgé pouvait affronter un séjour en réanimation. " Cohérent », parce que chez les patients âgés la « gestion des comorbidités » sera tout aussi importante pour permettre au patient de quitter l'hôpital et de s'en tenir éloigné.
Combien de temps pourra-t-on encore engager des procédures de soins lourdes et coûteuses chez des patients vulnérables qui pourraient en bénéficier, sans envisager une prise en charge globale et continue ? Comment justifier un séjour coûteux en réanimation, une chirurgie complexe, si c'est pour laisser le patient âgé au lit, perdre son autonomie en décompensant ses comorbidités au décours ? Mais à l'opposé, combien de temps continuera-t-on de refuser un patient sur le seul critère de l'âge, alors qu'il est en capacité d'affronter un séjour en réanimation, quand on accepte des patients plus jeunes avec des perspectives sombres à très court terme?

Finalement, seule l'organisation du parcours de soins doit permettre d'ouvrir les portes de la réanimation au patient âgé : 1) en l'évaluant avant ;2) en répondant rapidement à l'aval de la réanimation; et 3) en poursuivant la prise en charge urgente et complexe de la multimorbidité, ainsi que le maintien de l'autonomie au décours. Cette approche est rendue nécessaire par les considérations éthiques, mais aussi par les considérations économiques. Il est urgent de lancer des projets associant tous les acteurs de ces filières à venir, avec une évaluation de l'efficacité autant que de l'efficience, en utilisant des critères correspondant à la population gériatrique, comme l'absence de réhospitalisation précoce ou à moyen terme, la préservation de l'autonomie et de la qualité de vie à plus ou moins long terme. Ne pas le faire nous exposera à continuer de faire de l'âgisme, où du seul fait de l'âge, de l'heure, du dernier lit disponible, mais aussi de l'autocensure du médecin adresseur, des patients ne pourront bénéficier d'une prise en charge à laquelle ils ont droit et, surtout, qu'ils auraient été en mesure d'affronter.

Liens d'intérêts : Les auteurs déclarent ne pas avoir de lien d'intérêt.

\section{Références}

1. Population Division Department of Economic and Social Affairs (2001) World Population Ageing: 1950-2050 http://www.un.org/ esa/population/publications/worldageing19502050/index.htm

2. Bagshaw SM, Webb SAR, Delaney A, et al (2009) Very old patients admitted to intensive care in Australia and New Zealand: a multi-centre cohort analysis. Crit Care 13:R45

3. Nielsson MS, Christiansen CF, Johansen MB, et al (2014) Mortality in elderly ICU patients: a cohort study. Acta Anaesthesiol Scand 58:19-26

4. Conti M, Merlani P, Ricou B (2012) Prognosis and quality of life of elderly patients after intensive care. Swiss Med Wkly 142: w13671

5. Boddaert J, Cohen-Bittan J, Khiami F, et al (2014) Postoperative admission to a dedicated geriatric unit decreases mortality in elderly patients with hip fracture. PloS One 9:e83795

6. Somme D, Maillet JM, Gisselbrecht M, et al (2003) Critically ill old and the oldest-old patients in intensive care: short- and longterm outcomes. Intensive Care Med 29:2137-43 
7. De Rooij SE, Govers A, Korevaar JC, et al (2006) Short-term and long-term mortality in very elderly patients admitted to an intensive care unit. Intensive Care Med 32:1039-44

8. Fuchs L, Chronaki CE, Park S, et al (2012) ICU admission characteristics and mortality rates among elderly and very elderly patients. Intensive Care Med 38:1654-61

9. Sprung CL, Artigas A, Kesecioglu J, et al (2012) The Eldicus prospective, observational study of triage decision making in European intensive care units. Part II: intensive care benefit for the elderly. Crit Care Med 40:132-8
10. Zampieri FG, Colombari F (2014) The impact of performance status and comorbidities on the short-term prognosis of very elderly patients admitted to the ICU. BMC Anesthesiol 14:59

11. Boumendil A, Angus DC, Guitonneau AL, et al (2012) Variability of intensive care admission decisions for the very elderly. PloS One. 7:e34387

12. Le Maguet P, Roquilly A, Lasocki S, et al (2014) Prevalence and impact of frailty on mortality in elderly ICU patients: a prospective, multicenter, observational study. Intensive Care Med 40:674-82 